\title{
Maize (Zea mays L.) landraces classified by drought stress tolerance at the seedling stage
}

\author{
Víctor A. González-Hernández', Eleazar Lugo-Cruz' ${ }^{1 *}$ Leopoldo E. Mendoza-Onofre², \\ Amalio Santacruz-Varela ${ }^{3}$, Ma. Alejandra Gutiérrez-Espinosa ${ }^{4}$, Francisco Zavala-García ${ }^{5}$ \\ Departamentos de ${ }^{1}$ Fisiología Vegetal, ${ }^{2}$ Producción de Semillas, ${ }^{3}$ Genética, y ${ }^{4}$ Fruticultura, Colegio de Postgraduados, Campus Montecillo. \\ Carretera México-Texcoco km 36.5, 56230 Montecillo, Texcoco, Estado de México, México, ${ }^{5}$ Departamento de Fisiología Vegetal y Genética, \\ Facultad de Agronomía, Universidad Autónoma de Nuevo León. 66050 General Escobedo, Nuevo León, México
}

\section{A B S TR A C T}

Drought is the main limiting factor for maize production, and climate change can aggravate this water scarcity. One way to mitigate this problem is to plant drought tolerance maize genotypes. In landrace maize grown under rainfed conditions there are drought-adapted genotypes, which can be used in breeding programs for drought tolerance. The objective of this study was to evaluate the effect of an early water deficit on the seedling growth of 41 maize landraces from Nuevo León, Mexico, plus seven varieties, by means of drought tolerance indices based on biomass accumulation during both stress and post-stress recovery period, for identifying tolerant and susceptible genotypes. This study was performed at 2016 in Texcoco, Mexico $\left(19^{\circ} 27^{\prime} \mathrm{N}, 98^{\circ} 54^{\prime} \mathrm{W}, 2241\right.$ masl). In the greenhouse, 96 treatments were compared (48 genotypes $\times$ two soil water regimes: without and with drought) under randomized complete blocks experimental design. After the drought stress period, normal irrigation was resumed for 15 days for recovery. In maize landraces there is genetic diversity in drought tolerance. Landraces GalTrini and SITexas outstanded as the most water deficit tolerant, whereas landraces Berrones, Rodeo, Sabanilla, Carmen, AraTrini and the inbred line L65 were the most drought susceptible. The total biomass measured before water stress was not related to drought adaptability. This study demonstrates that the post stress recovery is more important in drought stress adaptability than the drought resistance, regarding root biomass, shoot biomass and total biomass. Thus, to include the post stress recovery in drought tolerance studies can produce a more precise genotypic classification for drought stress resistance and adaptability.

Keywords: Biomass seedling; Drought tolerance indices; Water deficit; Zea mays

\section{INTRODUCTION}

Maize (Zea mays L.) is one of the most important crops in the world. Maize growth, development and grain yield is frequently affected by drought in arid and semi-arid regions (Efeoğlu et al., 2009). The global warming will increase drought and will decrease crop productivity across the world. One way to prevent this problem is to grow maize genotypes tolerant to water deficit derived from breeding programs committed on increasing drought tolerance in the best landraces adapted to the region (Hellin et al., 2014). Maize landraces have genetic diversity that can be used in genetic breeding programs for drought tolerance, especially in genotypes adapted to rainfed, arid and semi-arid conditions.

Under rainfed conditions maize seedlings must survive the drought periods until rain resume; seedling death in this dry period generates low plant density and grain yield losses comparable to yield losses caused by drought at the blooming stage (Teruel et al., 2008). Therefore, in arid and semi-arid climates, both drought resistance and drought recovery are key determinants in crop drought adaptability (Perrone et al., 2012). Plant drought adaptability is the comprehensive capacity integrating both drought resistance (drought escape, drought avoidance or drought tolerance), and drought recovery after the water stress period, when the watering is resuming (Chen et al., 2016).

Plant responses to water deficit are complex because they depend on drought severity and duration, as well as on plant development stage and genotype (Aslam et al., 2015). For example, for maize seedlings it has been reported that water deficit produces reductions in cell turgor, cell division and cell enlargement (Anjorin et al., 2017); consequently,

\footnotetext{
*Corresponding author:

Eleazar Lugo-Cruz, Departamentos de Fisiología Vegetal, Colegio de Postgraduados, Campus Montecillo. Carretera México-Texcoco km 36.5, 56230 Montecillo, Texcoco, Estado de México, México.
} 
drought may modify the root/shoot biomass ratio, and decrease gas exchange and assimilated storage capacity (Chen et al., 2016; Akinwale et al., 2017). The genetic diversity available within the maize genotypes has allowed to find genotypes having the ability to prevent growth losses induced by drought at the seedling stage (Mabhaudhi and Modi, 2010; Bashir et al., 2016; Anjorin et al., 2017; Badr et al., 2020; Pawar et al., 2020), while other researchers have reported tolerant species populations (including maize) having the ability to compensate the growth losses induced by drought (Xu et al., 2010; Chen et al., 2016). Therefore, it is postulated here that it should be possible to find maize genotypes adapted to dry environments having both drought tolerance during the stress and an adequate post-stress recovery at the seedling stage.

Drought tolerant maize seedlings must grow quickly and have a high water use efficiency for biomass production, as well as capacity to form roots for absorbing water at low edaphic water potential or greater soil depth. According to Richards et al. (2002), vigorous seedlings quickly cover the soil with foliage; theses seedlings will have increased crop competitiveness for intercepting photosynthetic active radiation and for water use efficiency.

Then, a high biomass accumulation at the early vegetative stage is a promising feature to select vigorous maize genotypes for drought stress tolerance, most specially if this tolerance is maintained at different growth stages (Bashir et al., 2016). In this context, the objective of the present study was to evaluate the effect of an water deficit on the seedling growth of 41 Mexican maize landraces, by using several drought tolerance indices based on biomass accumulation and distribution during both the stress and the post-stress recovery periods, for identifying drought tolerant genotypes, and the main tolerance mechanisms.

\section{MATERIALS AND METHODS}

\section{Experimental site and plant material}

The study was carried out in Texcoco, Mexico (19 ${ }^{\circ} 27^{\prime}$ $\left.\mathrm{N}, 98^{\circ} 54^{\prime} \mathrm{W}, 2241 \mathrm{masl}\right)$ under greenhouse conditions. In 2016, 48 maize genotypes were evaluated, of which 41 were maize landraces native from Southern Nuevo Leon state, Mexico (Fig. 1). In this region the maize landraces are commonly grown under rainfed conditions, at altitudes between 1049 and 2619 m (INEGI, 2019). Five experimental inbred lines (L22, L65, L69, L104 and L134) developed in the Central Valleys of Mexico as part of the "Maize Ideotype Formation Project" at the Colegio de Postgraduados, Mexico, were used as control varieties, plus two Mexican commercials hybrids (HS2 and Promesa) generated by the same institution.

\section{Treatments and experimental design}

The 96 treatments (48 genotypes $\times 2$ soil water regimes) were established under a randomized complete blocks design with four replications. The experimental unit consisted of four seedlings grown individually in styrofoam vases $(1 \mathrm{~L})$ filled with $700 \mathrm{~g}$ of substrate (soil and peat moss at 2:1 ratio). Sowing was done from September 29 to October 2, 2016 (one repetition per day). Two seeds were sown per pot, and 12 days after sowing (das) the seedlings were thinned to one per pot. The two soil water regimes imposed were: 1) Control, with soil moisture maintained between 50 and $100 \%$ of field capacity during 56 das; and 2) Drought, by withholding watering during 12 days (from 29 to 41 das) so that soil humidity declined to $10 \%$, below the permanent wilting point (16\%). At 41 das irrigation was restored to the control level and then maintained for 15 days (Fig. 2). Soil moisture percentage was determined by gravimetric method in 52 pots per each water regime, based on the daily water losses (weight losses).

\section{Growth analyses}

Following the basic procedures of Hunt (1990) for plant growth analysis, the biomass of $\operatorname{root}(\mathrm{RB}, \mathrm{g})$, $\operatorname{shoot}(\mathrm{SB}, \mathrm{g})$ and total $(\mathrm{TB}=\mathrm{RB}+\mathrm{SB}, \mathrm{g})$ were measured in each seedling of the 4 replications, at three stages: initiation of drought stress, end of drought stress, and end of recovery. RB and SB were recorded after drying roots and shoots separately at $70^{\circ} \mathrm{C}$ in a forced convection drying oven (Riossa, model HCFD-82, Mexico) until constant weight. All samples were weighed on digital analytical balance (Sartorius Handy H51, Germany). The root/shoot ratio (R/S) was calculated as $\mathrm{RB} / \mathrm{SB}$ in drought treatment at the end of drought stress (DE) and at the end of recovery (RE).

\section{Drought stress tolerance indices}

Nine indices were calculated as follows: three for drought resistance (ResRB, ResSB and ResTB), three for drought recovery (RecRB, RecSB and RecTB), and three for drought adaptability (AdapRB, AdapSB and AdapTB). These indices were estimated based on the root (RB), shoot (SB) and total (TB) biomass accumulation, according to the equations proposed by Chen et al. (2016):

$$
\begin{aligned}
& \begin{array}{l}
\text { Drought resistance } \\
(\operatorname{Res} R B, \operatorname{Res} S B \text { and ResTB })
\end{array}=\frac{B D E_{\text {drought }}-B D I_{\text {drought }}}{B D E_{\text {control }}-B D I_{\text {control }}}(\text { Eq. 1) } \\
& \begin{array}{l}
\text { Drought recovery } \\
(\operatorname{Rec} R B, \operatorname{Rec} S B \text { and } \operatorname{RecTB})
\end{array}=\frac{B R E_{\text {drought }}-B D E_{\text {drought }}}{B R E_{\text {control }}-B D E_{\text {control }}}(\text { Eq. 2) }
\end{aligned}
$$$$
\text { Drought adaptability }_{(\text {dapR } B, A d a p S B \text { and } A d a p T B)}=\frac{B R E_{\text {drought }}-B D I_{\text {drought }}}{B R E_{\text {control }}-B D I_{\text {control }}}
$$ 


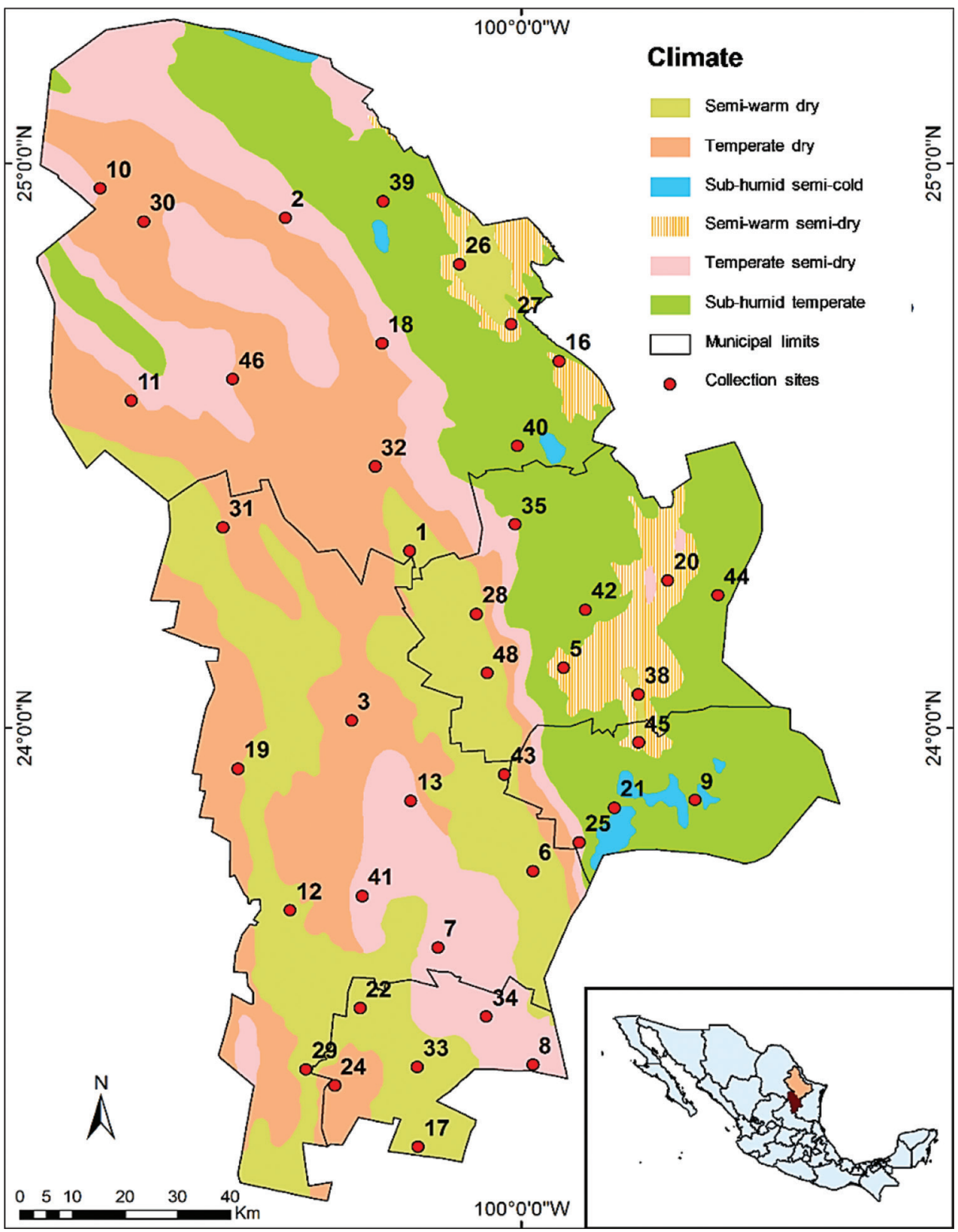

Fig 1. Geographical distribution of maize entries (Zea mays L.) from Southern Nuevo Leon state, Mexico. Map and climates taken from INEGI (2019). The numbers correspond to the collection site of each maize entry that is described in Table 1.

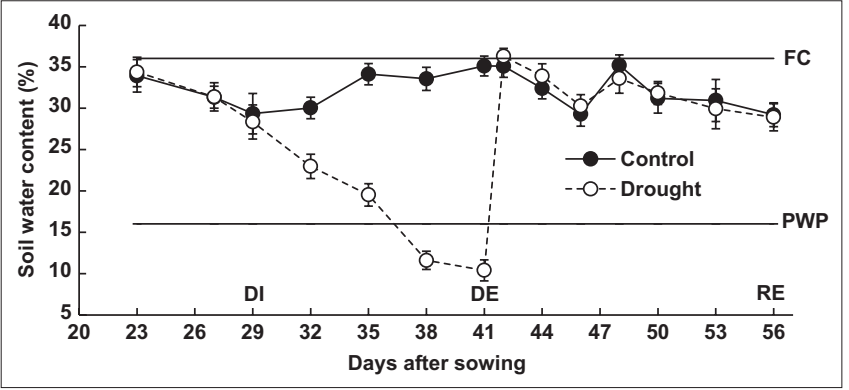

Fig 2. Changes in soil water content during the drought stress and the recovery periods. $\mathrm{FC}=$ Field capacity. $\mathrm{PWP}=$ Permanent wilting point. $\mathrm{DI}$ and $\mathrm{DE}=$ Initiation and end of drought stress. $\mathrm{RE}=\mathrm{End}$ of recovery. Vertical lines in each observation point correspond to standard deviation.
Where: $\mathrm{BDI}=$ biomass $(\mathrm{RB}, \mathrm{SB}$ or $\mathrm{TB})$ at initiation of drought stress (29 das); BDE = biomass $(\mathrm{RB}, \mathrm{SB}$ or $\mathrm{TB})$ at the end of drought stress (41 das); BRE = biomass (RB, $\mathrm{SB}$ or $\mathrm{TB})$ at the end of the recovery period (56 das).

The maize genotypes were classified as tolerant or susceptible based on the drought adaptability index calculated with total biomass (AdapBT), with data registered at the end of the experiment (water stress period + recovery period). The other indices (drought resistance and drought recovery) calculated for root, shoot and total biomass were used to find out the evaluation period when most of the biomass production occurred during the two 
experimental periods: the drought stress period or the drought recovery period.

\section{Statistical analyses}

Data were submitted to analyses of variance using SAS 9.4 statistical package (SAS Institute, 2015). Multiple comparison tests were made with least significant difference (LSD, $\mathrm{p} \leq 0.05$ ), which allowed to identify the tolerant genotypes by means total biomass lowest difference between drought and control treatments. Pearson's correlation coefficients between drought tolerance indices, total biomass at initiation of drought stress and the $\mathrm{R} / \mathrm{S}$ ratio in drought conditions were estimated using PROC CORR procedure in SAS 9.4 statistical package ( $\mathrm{p} \leq 0.05)$.

\section{RESULTS}

For total biomass (TB) eight native maize landraces (Refugio, Delicias, Escondida, Carpintería, Catorce, Salero, Tomates, SJDios) and one inbred line (L134) showed high drought resistance indexes (ResTB), with values above 1.0 (ResTB $=1.18$ to 2.16), because at the end of the drought period they had higher total biomass than in their irrigated controls ( $\mathrm{p} \leq 0.05)$, as shown in Table 1. On the contrary, six landraces (SLázaro, Lagunita, Siberia, Sacramento, SGrande, Carmen), and two inbred lines (L104 and L65) had low drought resistance indexes (ResTB $=0.30$ to 0.81 ), since they had lost total biomass during the drought period compared to their controls $(\mathrm{p} \leq 0.05)$.

For total biomass (RecTB) measured at the post-stress recovery period, seven native landraces (Siberia, RCedillo, GalTrini, Sacramento, SITexas, Altitos, Hediondilla) and two inbred lines (L22 and L104) accumulated more biomass after the recovery period than their irrigated controls $(\mathrm{p} \leq 0.05)$, with recovery indexes greater than 1.0 $(\operatorname{RecTB}=1.2$ to 3.0). In this group, landraces Siberia and Sacramento stood out because they were able to recover the biomass lost during water stress, so that at the end of the recovery period their total biomass was the same as in their controls growing in normal irrigation (Table 1). In contrast, 15 landraces (Escondida, Cardona, SJDios, Tomates, Catorce, Delicias, Lindero, Carpintería, Crucitas, SAnita, PAnteojitos, Berrones, Rodeo, Sabanilla, AraTrini) and the inbred line L65, had the lowest recovery indexes with negative values (RecTB $=-1.42$ to -0.07 ).

Regarding the adaptability analysis for total biomass (AdapTB $=$ drought resistance + drought recovery), six landraces (SITexas, GalTrini, RCedillo, Escondida, SAPNevada and Altitos), and the inbred line L22 showed the highest drought adaptability indexes with values higher than 1.0 (AdapTB $=1.08$ to 1.41). In contrast, 41 maize genotypes (35 landraces and six control varieties) showed drought adaptability indexes lesser than 1.0 (Table 1). Among these susceptible genotypes, 22 also showed less total biomass $(p \leq 0.05)$ at the end of the whole evaluation (15 days after rewatering, 56 das).

Correlation analysis showed 22 positive and nine negative associations $(p \leq 0.05)$ (Table 2). The strongest positive associations $(\mathrm{r}>0.66)$ were found between ResTB vs. $\operatorname{ResRB}(r=0.79)$ and ResSB $(r=0.74)$, RecTB vs. RecRB $(\mathrm{r}=0.78)$, and AdapTB $v$. AdapRB $(\mathrm{r}=0.87)$ and AdapSB $(\mathrm{r}=0.67)$.

\section{DISCUSSION}

The maize landraces collected in rainfed regions of Southern Nuevo Leon state, Mexico, showed a wide diversity in drought stress adaptability. This genetic diversity allowed identifying drought stress tolerant and drought stress susceptible genotypes. At the end of the present study (drought stress period + recovery cycle, 56 das), 26 genotypes showed no significant decreases in total biomass even though they were submitted to a rather severe water deficit in which the soil moisture became diminished $6 \%$ below the permanent wilting point $(16 \%)$.

Landraces GalTrini and SITexas stood out as the most drought tolerant in this test, because their seedlings were able to keep growing and to accumulate more total biomass $(p \leq 0.05)$ at the end of the whole evaluation (56 das) than their irrigated controls, and they were also able to maintain a balanced biomass distribution since their root and shoot ratio were near to $1.0(\mathrm{R} / \mathrm{S}-\mathrm{RE}=1.1$ and 1.0 , respectively). These two outstanding genotypes combine two mechanisms for drought stress tolerance: 1) They did not lose biomass during the water stress period, and 2) They were able to resume plant growth and biomass accumulation after the stress, during the recovery period, with gains of 115 and $75 \%$, respectively. Therefore, these tolerant/adaptable genotypes have seedlings that either avoided or tolerated tissue dehydration, physiological responses that according to Turner (1979) are due to maintaining cell turgor and elasticity during the stress by osmotic adjustment.

On the contrary, 22 genotypes were classified as drought susceptible because their adaptability indices were lesser than 1.0, so that at the end of the experiment (56 das) their total biomass was significantly lower than their irrigated controls. The most drought susceptible genotypes were five maize landraces (Berrones, Rodeo, Sabanilla, Carmen and AraTrini), and the inbred line L65, which showed the 
Table 1: Drought stress indexes calculated for root (RB), shoot (SB) and total (TB) biomass, root/shoot ratio (R/S) and total biomass comparison of 48 maize genotypes grown under drought stress (Drought) and irrigated conditions (Control)

\begin{tabular}{|c|c|c|c|c|c|c|c|c|c|c|c|}
\hline \multirow[t]{3}{*}{ No. } & \multirow[t]{3}{*}{ Genotype } & \multicolumn{5}{|c|}{ Drought stress index $(\mathrm{g} / \mathrm{g})$} & \multicolumn{5}{|c|}{ Total biomass (g/plant) } \\
\hline & & \multicolumn{3}{|c|}{ Total biomass } & \multicolumn{2}{|c|}{ R/S } & \multirow[t]{2}{*}{ DI } & \multicolumn{2}{|c|}{ DE } & \multicolumn{2}{|c|}{ RE } \\
\hline & & ResTB & RecTB & AdapTB & $\mathrm{DE}$ & RE & & Control & Drought & Control & Drought \\
\hline 1 & SITexas & 1.17 & 1.75 & 1.41 & 0.9 & 1.0 & $0.560^{\mathrm{h}-\mathrm{mD}}$ & $0.702^{\mathrm{C}}$ & $0.726^{\mathrm{BC}}$ & $0.803^{\mathrm{vwB}}$ & $0.904^{\mathrm{j}-\mathrm{rA}}$ \\
\hline 2 & GalTrini & 1.08 & 2.15 & 1.31 & 1.3 & 1.1 & $0.574^{\mathrm{g}-\mathrm{kD}}$ & $0.909^{C}$ & $0.934^{\mathrm{BC}}$ & $1.000^{i-s B}$ & $1.131^{\mathrm{abA}}$ \\
\hline 3 & RCedillo & 0.66 & 2.81 & 1.25 & 1.0 & 1.0 & $0.640^{\mathrm{b}-\mathrm{eD}}$ & $0.878^{\mathrm{BC}}$ & $0.797^{c}$ & $0.970^{1-\mathrm{AAB}}$ & $1.054^{\mathrm{b}-\mathrm{AA}}$ \\
\hline 4 & L22 & 0.95 & 2.42 & 1.22 & 0.9 & 0.9 & $0.384^{\mathrm{vc}}$ & $0.707^{B}$ & $0.692^{\mathrm{B}}$ & $0.777^{\text {WAB }}$ & $0.861^{\text {n-uA }}$ \\
\hline 5 & Escondida & 1.66 & -0.33 & 1.20 & 0.9 & 0.9 & $0.498^{\mathrm{o}-\mathrm{uc}}$ & $0.784^{\mathrm{B}}$ & $0.974^{\mathrm{A}}$ & $0.872^{t-w A B}$ & $0.945^{f-n A}$ \\
\hline 6 & SAPNevada & 1.09 & 0.23 & 1.18 & 0.9 & 0.8 & $0.507^{n-u c}$ & $0.989^{\mathrm{AB}}$ & $1.035^{\mathrm{A}}$ & $0.946^{n-t B}$ & $1.025^{\mathrm{b}-\mathrm{AA}}$ \\
\hline 7 & Altitos & 0.89 & 1.41 & 1.08 & 1.4 & 1.5 & $0.598^{\mathrm{e}-\mathrm{iD}}$ & $0.884^{\mathrm{BC}}$ & $0.853^{c}$ & $1.048^{f-O A B}$ & $1.084^{\mathrm{a}-\mathrm{dA}}$ \\
\hline 8 & Cardona & 1.15 & -0.18 & 0.99 & 1.2 & 1.2 & $0.508^{n-t B}$ & $0.769^{A}$ & $0.807^{A}$ & $0.803^{\mathrm{vwA}}$ & $0.801^{q-w A}$ \\
\hline 9 & Refugio & 2.16 & 0.26 & 0.98 & 1.1 & 0.8 & $0.498^{\mathrm{o}-\mathrm{uc}}$ & $0.665^{\mathrm{B}}$ & $0.858^{A}$ & $0.937^{0-4 A}$ & $0.930^{g-p A}$ \\
\hline 10 & Hediondilla & 0.78 & 1.20 & 0.98 & 0.6 & 0.7 & $0.585^{f-j C}$ & $0.859^{B}$ & $0.799^{B}$ & $1.115^{\mathrm{chA}}$ & $1.106^{\mathrm{a}-\mathrm{cA}}$ \\
\hline 11 & SJDios & 1.18 & -0.86 & 0.97 & 1.1 & 1.1 & $0.568^{\text {h-1C }}$ & $0.894^{\mathrm{B}}$ & $0.953^{A}$ & $0.932^{p-u A B}$ & $0.920^{h-p A B}$ \\
\hline 12 & SGregorio & 1.10 & 0.79 & 0.96 & 1.1 & 1.1 & $0.541^{k-p C}$ & $0.803^{\mathrm{B}}$ & $0.828^{B}$ & $0.996^{\mathrm{j}-\mathrm{sA}}$ & $0.980^{\mathrm{d}-\mathrm{mA}}$ \\
\hline 13 & SantaMa & 1.05 & 0.71 & 0.93 & 0.9 & 0.9 & $0.525^{1-\mathrm{rC}}$ & $0.838^{\mathrm{B}}$ & $0.852^{\mathrm{B}}$ & $0.994^{j-s A}$ & $0.963^{e-n A}$ \\
\hline 14 & L69 & 1.05 & 0.44 & 0.92 & 0.7 & 0.7 & $0.544^{\mathrm{j}-\mathrm{oB}}$ & $0.829^{A}$ & $0.844^{\mathrm{A}}$ & $0.905^{r-v A}$ & $0.877^{\mathrm{m}-\mathrm{uA}}$ \\
\hline 15 & L134 & 1.75 & 0.36 & 0.91 & 0.7 & 0.8 & $0.525^{1-r D}$ & $0.734^{\mathrm{C}}$ & $0.891^{\mathrm{B}}$ & $1.056^{f-n A}$ & $1.009^{\mathrm{c}-\mathrm{kA}}$ \\
\hline 16 & Tomates & 1.46 & -0.64 & 0.91 & 1.3 & 0.9 & $0.505^{\mathrm{o}-\mathrm{uc}}$ & $0.852^{B}$ & $1.001^{\mathrm{A}}$ & $0.967^{1-\mathrm{tA}}$ & $0.927^{g-p A B}$ \\
\hline 17 & Sanlsidro & 1.25 & 0.37 & 0.89 & 1.1 & 1.1 & $0.649^{b-d c}$ & $0.897^{\mathrm{B}}$ & $0.960^{\mathrm{AB}}$ & $1.067^{\mathrm{e}-\mathrm{mA}}$ & $1.022^{\mathrm{b}-\mathrm{iA}}$ \\
\hline 18 & Tokio & 1.01 & 0.81 & 0.88 & 0.9 & 0.8 & $0.475^{\mathrm{s}-\mathrm{uB}}$ & $0.639^{\mathrm{B}}$ & $0.640^{\mathrm{B}}$ & $0.955^{\mathrm{m}-\mathrm{A}}$ & $0.897^{\mathrm{k}-\mathrm{sA}}$ \\
\hline 19 & Yerba & 0.95 & 0.79 & 0.88 & 1.0 & 1.1 & $0.605^{d-h c}$ & $0.891^{\mathrm{B}}$ & $0.876^{\mathrm{B}}$ & $1.127^{\mathrm{c}-\mathrm{gA}}$ & $1.063^{\mathrm{b}-\mathrm{eA}}$ \\
\hline 20 & Sacramento & 0.58 & 1.93 & 0.87 & 0.8 & 0.9 & $0.521^{\mathrm{m}-\mathrm{sc}}$ & $0.794^{A}$ & $0.679^{B}$ & $0.871^{\text {t-wA }}$ & $0.826^{\mathrm{o}-\mathrm{VA}}$ \\
\hline 21 & Siberia & 0.58 & 3.00 & 0.85 & 0.7 & 0.7 & $0.663^{\mathrm{bcc}}$ & $1.212^{\mathrm{A}}$ & $0.984^{\mathrm{B}}$ & $1.280^{\mathrm{abA}}$ & $1.187^{\mathrm{aA}}$ \\
\hline 22 & Caminos & 1.30 & 0.45 & 0.85 & 1.0 & 0.9 & $0.521^{\mathrm{m}-\mathrm{tc}}$ & $0.723^{\mathrm{B}}$ & $0.784^{\mathrm{B}}$ & $0.954^{\mathrm{m}-\mathrm{tA}}$ & $0.888^{1-t A}$ \\
\hline 23 & HS2 & 1.00 & 0.25 & 0.84 & 0.9 & 0.9 & $0.676^{\mathrm{bB}}$ & $1.010^{\mathrm{A}}$ & $1.010^{\mathrm{A}}$ & $1.101^{\mathrm{C}-\mathrm{jA}}$ & $1.033^{\mathrm{b}-\mathrm{hA}}$ \\
\hline 24 & TaponaMo & 0.77 & 0.86 & 0.80 & 1.1 & 1.0 & $0.474^{\mathrm{tuD}}$ & $0.858^{\mathrm{C}}$ & $0.771^{c}$ & $1.088^{\mathrm{d}-\mathrm{kA}}$ & $0.968^{\mathrm{e}-\mathrm{nB}}$ \\
\hline 25 & SLázaro & 0.81 & 0.41 & 0.78 & 1.0 & 1.0 & $0.597^{\mathrm{e}-\mathrm{iC}}$ & $1.042^{\mathrm{A}}$ & $0.959^{B}$ & $1.085^{\mathrm{d}-\mathrm{kA}}$ & $0.977^{\mathrm{d}-\mathrm{mB}}$ \\
\hline 26 & BJalisco & 0.75 & 0.79 & 0.78 & 1.2 & 0.9 & $0.493^{q-u c}$ & $0.708^{\mathrm{B}}$ & $0.655^{\mathrm{B}}$ & $1.004^{\mathrm{h}-\mathrm{rA}}$ & $0.889^{1-\mathrm{tA}}$ \\
\hline 27 & Poza & 1.01 & 0.69 & 0.77 & 1.1 & 1.1 & $0.487^{q-u B}$ & $0.584^{\mathrm{B}}$ & $0.585^{\mathrm{B}}$ & $0.887^{\text {s-wA }}$ & $0.794^{r-w A}$ \\
\hline 28 & SGrande & 0.56 & 0.95 & 0.74 & 1.2 & 1.1 & $0.516^{\mathrm{m}-\mathrm{tE}}$ & $0.813^{C}$ & $0.684^{\mathrm{D}}$ & $1.058^{f-n A}$ & $0.916^{\mathrm{i}-\mathrm{qB}}$ \\
\hline 29 & Catorce & 1.60 & -1.42 & 0.73 & 1.1 & 1.1 & $0.685^{\mathrm{bc}}$ & $0.989^{\mathrm{B}}$ & $1.173^{\mathrm{A}}$ & $1.112^{\mathrm{c}-\mathrm{A} A}$ & $0.998^{\mathrm{C}-1 \mathrm{~B}}$ \\
\hline 30 & Delicias & 1.76 & -0.70 & 0.73 & 0.9 & 0.8 & $0.407^{v C}$ & $0.651^{B}$ & $0.835^{A}$ & $0.826^{\mathrm{u}-\mathrm{wA}}$ & $0.713^{v-\times B}$ \\
\hline 31 & Boquilla & 0.83 & 0.26 & 0.72 & 1.2 & 1.1 & $0.569^{h-k c}$ & $0.978^{\mathrm{B}}$ & $0.909^{B}$ & $1.076^{\mathrm{d}-\mathrm{A}}$ & $0.935^{\mathrm{g}-\mathrm{OB}}$ \\
\hline 32 & Salero & 1.56 & 0.02 & 0.72 & 1.0 & 1.2 & $0.606^{d-h D}$ & $0.865^{c}$ & $1.009^{B}$ & $1.179^{\mathrm{b}-\mathrm{eA}}$ & $1.017^{\mathrm{b}-\mathrm{jB}}$ \\
\hline 33 & Lindero & 0.99 & -0.07 & 0.67 & 1.1 & 1.2 & $0.602^{\mathrm{e}-\mathrm{hc}}$ & $0.939^{\mathrm{B}}$ & $0.936^{\mathrm{B}}$ & $1.088^{\mathrm{d}-\mathrm{kA}}$ & $0.926^{g-p B}$ \\
\hline 34 & LTaberna & 0.84 & 0.48 & 0.66 & 1.1 & 1.5 & $0.529^{k-q D}$ & $0.858^{\mathrm{BC}}$ & $0.806^{c}$ & $1.180^{\mathrm{b}-\mathrm{dA}}$ & $0.960^{\mathrm{e}-\mathrm{nB}}$ \\
\hline 35 & Ánimas & 0.55 & 0.79 & 0.66 & 1.0 & 1.1 & $0.616^{d-g C}$ & $0.867^{A B}$ & $0.754^{\mathrm{BC}}$ & $1.071^{\mathrm{d}-\mathrm{A}}$ & $0.915^{i-q A B}$ \\
\hline 36 & Promesa & 0.70 & 0.53 & 0.63 & 0.8 & 0.9 & $0.629^{c-f c}$ & $1.045^{\mathrm{B}}$ & $0.919^{\mathrm{B}}$ & $1.314^{\mathrm{aA}}$ & $1.062^{\mathrm{b}-\mathrm{eB}}$ \\
\hline 37 & L104 & 0.30 & 1.44 & 0.63 & 0.6 & 0.7 & $0.754^{\mathrm{ac}}$ & $1.079^{\mathrm{AB}}$ & $0.852^{\mathrm{C}}$ & $1.207^{\mathrm{a}-\mathrm{cA}}$ & $1.037^{\mathrm{b}-\mathrm{gB}}$ \\
\hline 38 & Carpintería & 1.63 & -0.85 & 0.59 & 1.4 & 1.0 & $0.482^{r-u C}$ & $0.736^{\mathrm{B}}$ & $0.896^{A}$ & $0.919^{p-u A}$ & $0.741^{v-x B}$ \\
\hline 39 & Lagunita & 0.62 & 0.32 & 0.57 & 1.0 & 1.1 & $0.492^{q-u c}$ & $0.941^{A}$ & $0.769^{B}$ & $1.019^{g-q A}$ & $0.794^{r-w B}$ \\
\hline 40 & Crucitas & 0.77 & -0.60 & 0.55 & 1.0 & 1.1 & $0.552^{\mathrm{i}-\mathrm{nD}}$ & $0.911^{A B}$ & $0.827^{\mathrm{BC}}$ & $0.979^{k-t A}$ & $0.786^{s-w c}$ \\
\hline 41 & SAnita & 1.34 & -0.73 & 0.54 & 1.2 & 1.0 & $0.460^{\mathrm{uD}}$ & $0.761^{\mathrm{BC}}$ & $0.865^{\mathrm{AB}}$ & $0.952^{n-t A}$ & $0.725^{\mathrm{v}-\mathrm{xc}}$ \\
\hline 42 & PAnteojitos & 1.08 & -0.09 & 0.51 & 1.5 & 1.2 & $0.529^{k-p c}$ & $0.774^{\mathrm{B}}$ & $0.792^{\mathrm{B}}$ & $1.007^{\mathrm{h}-\mathrm{rA}}$ & $0.772^{\mathrm{u}-\mathrm{wB}}$ \\
\hline 43 & Berrones & 1.19 & -0.41 & 0.48 & 0.9 & 1.0 & $0.486^{q-u c}$ & $0.737^{\mathrm{B}}$ & $0.785^{\mathrm{B}}$ & $0.939^{\circ-4 A}$ & $0.701^{w \times B}$ \\
\hline 44 & Rodeo & 0.74 & -0.08 & 0.47 & 1.0 & 0.8 & $0.498^{\circ-u C}$ & $0.813^{\mathrm{B}}$ & $0.732^{\mathrm{B}}$ & $0.967^{1-\mathrm{tA}}$ & $0.719^{v-x B}$ \\
\hline 45 & Sabanilla & 1.54 & -0.17 & 0.41 & 1.3 & 1.4 & $0.596^{\mathrm{e}-\mathrm{iC}}$ & $0.743^{\mathrm{B}}$ & $0.824^{\mathrm{B}}$ & $1.032^{\mathrm{g}-\mathrm{pA}}$ & $0.775^{\mathrm{t}-\mathrm{wB}}$ \\
\hline 46 & Carmen & 0.37 & 0.39 & 0.38 & 1.1 & 1.2 & $0.497^{\mathrm{p}-\mathrm{uD}}$ & $0.787^{\mathrm{B}}$ & $0.605^{\mathrm{CD}}$ & $0.913^{q-v A}$ & $0.655^{x C}$ \\
\hline 47 & L65 & 0.65 & -0.91 & 0.37 & 0.7 & 0.8 & $0.623^{c-f C}$ & $1.055^{A}$ & $0.902^{\mathrm{B}}$ & $1.149^{\mathrm{C}-\mathrm{fA}}$ & $0.816^{p-w B}$ \\
\hline 48 & AraTrini & 1.02 & -0.48 & 0.35 & 1.0 & 0.8 & $0.492^{q-u c}$ & $0.746^{\mathrm{BC}}$ & $0.751^{\mathrm{B}}$ & $0.954^{\mathrm{m}-\mathrm{tA}}$ & $0.651^{\times \mathrm{BC}}$ \\
\hline
\end{tabular}

Res, Rec and Adap = resistance, recovery and adaptability indexes calculated for total biomass (TB). DI and DE $=$ initiation and end of drought stress. RE $=$ end of recovery. Different capital letter in the same row to indicate statistical differences between evaluation stages in each genotype (DMS, $p \leq 0.05$ ). Different lowercase letters in the same column indicate statistical differences between genotypes (DMS, $p \leq 0.05$ ). Hyphen between lowercase letters represents letters that are arranged alphabetically between two letters shown 
Table 2: Pearson correlation coefficients between drought stress indices, root/shoot ratio and total biomass at initiation of drought stress

\begin{tabular}{|c|c|c|c|c|c|c|c|c|c|c|c|c|}
\hline & $\begin{array}{l}\text { Res } \\
\text { RB }\end{array}$ & $\begin{array}{l}\text { Rec } \\
\text { RB }\end{array}$ & $\begin{array}{c}\text { Adap } \\
\text { RB }\end{array}$ & $\begin{array}{l}\text { Res } \\
\text { SB }\end{array}$ & $\begin{array}{l}\text { Rec } \\
\text { SB }\end{array}$ & $\begin{array}{c}\text { Adap } \\
\text { SB }\end{array}$ & $\begin{array}{c}\text { Res } \\
\text { TB }\end{array}$ & $\begin{array}{c}\text { Rec } \\
\text { TB }\end{array}$ & $\begin{array}{c}\text { Adap } \\
\text { TB }\end{array}$ & $\begin{array}{l}R / S \\
D E\end{array}$ & $\begin{array}{l}R / S \\
-R E \\
\end{array}$ & $\begin{array}{l}\text { TB } \\
\text {-DI }\end{array}$ \\
\hline ResRB & 1.00 & & & & & & & & & & & \\
\hline RecRB & -0.20 & 1.00 & & & & & & & & & & \\
\hline AdapRB & $0.4033^{\star *}$ & $0.49^{* *}$ & 1.00 & & & & & & & & & \\
\hline ResSB & $0.30^{*}$ & -0.17 & 0.08 & 1.00 & & & & & & & & \\
\hline RecSB & $-0.30^{*}$ & 0.19 & 0.14 & $-0.45^{\star *}$ & 1.00 & & & & & & & \\
\hline AdapSB & -0.15 & $0.33^{*}$ & 0.25 & 0.24 & $0.44^{* *}$ & 1.00 & & & & & & \\
\hline ResTB & $0.79^{\star *}$ & $-0.29^{*}$ & 0.22 & $0.74^{\star *}$ & $-0.49^{* *}$ & -0.01 & 1.00 & & & & & \\
\hline RecTB & $-0.29^{*}$ & $0.78^{* *}$ & $0.46^{* *}$ & $-0.31^{*}$ & $0.45^{* *}$ & $0.46^{* *}$ & $-0.47^{* *}$ & 1.00 & & & & \\
\hline AdapTB & 0.24 & $0.49^{\star *}$ & $0.87^{* *}$ & 0.18 & $0.31^{*}$ & $0.67^{* *}$ & 0.19 & $0.54^{* *}$ & 1.00 & & & \\
\hline R/S-DE & $0.42^{* *}$ & -0.27 & -0.03 & 0.00 & -0.14 & -0.16 & $0.47^{\star *}$ & $-0.43^{\star *}$ & -0.09 & 1.00 & & \\
\hline R/S-RE & $0.37^{\star}$ & 0.24 & $0.52^{* *}$ & -0.11 & -0.10 & -0.07 & 0.26 & 0.06 & $0.37^{\star *}$ & $0.58^{* *}$ & 1.00 & \\
\hline TBDI & -0.07 & 0.13 & -0.04 & $-0.41^{* *}$ & 0.24 & 0.06 & -0.27 & 0.17 & 0.00 & -0.01 & 0.03 & 1.00 \\
\hline
\end{tabular}

ResRB, RecRB and AdapRB = resistance, recovery and adaptability indexes calculated with root biomass. ResSB, RecSB and AdapSB = resistance, recovery and adaptability indexes calculated with shoot biomass. ResTB, RecTB and AdapTB = resistance, recovery and adaptability indexes calculated with total biomass. TB-DI = total biomass at initiation of drought stress (g/plant). R/S-DE and R/S-RE = root/shoot ratios in the drought treatment calculated at the end of drought stress and at the end of drought recovery period. ${ }^{*}$ and ${ }^{* *}=$ statistical significance with $p \leq 0.05$ and $p \leq 0.01$, respectively

lowest drought adaptability indices, and had the larger losses in accumulated biomass that ranged between 52 and $65 \%$.

The observed differences in maize responses could be attributed to a the a large list of processes that other researchers have reported as negatively affected in maize, such as decreases in chlorophyll content, leaf transpiration, nutrient absorption, photosynthesis rate, cellular division and enlargement, while the reactive oxygen species synthesis become increased (López-Santillán et al., 2004; Mabhaudhi and Modi, 2010; Akinwale et al., 2017). Consequently, drought stress causes a reduced internode length and less ability to store assimilates, as well as losses in plant density and grain yield (Teruel et al., 2008). All these plant responses to water deficit depend on the drought severity and duration, on the plant development stage and on the genotype (Aslam et al., 2015).

Most importantly, in this study it is shown a rather large genetic variation among the studied maize landraces, regarding drought stress responses measured on biomass accumulation and plant growth, variation that was determined by the three measured indices: drought stress tolerance, drought recovery and drought adaptability. According to Gil et al. (2004), these genetic variations should be due to genetic differences in maize adaptation to dry conditions. It might be inferred then that the drought tolerance landraces should contain several tolerance genes (which remain to be identified), such as the Rav17 gene that codifies for a dehydrin associated to drought stress tolerance as recently found in Mexican maize (HernándezGuillén, 2016; Belmont-Valadez, 2018).

Several researchers have associated maize seedling biomass (early vigor) with grain yield, deep root system and fast growth that allow less water loss by evaporation, increased water use efficiency and better seedling growth (Munns and Richards, 2007; Chen et al., 2016; Trachsel et al., 2016). However, vigorous seedlings are not necessarily associated to drought tolerant plants, since the three drought adaptability indices were not correlated to the initial total biomass measured at the start of the drought stress period (AdapRB vs. TBDI [ $\mathrm{r}=-0.04 \mathrm{~ns}]$, AdapSB vs. TBDI $[\mathrm{r}=0.06 \mathrm{~ns}]$ and AdapBT vs. TBDI [ $\mathrm{r}=-0.001 \mathrm{~ns}]$ ), even when the maize genotype with the highest total biomass before the stress, TBDI (line L104) was almost twice as large as the two genotypes with the lowest TBDI genotypes (Line L22 and Delicias). Only one genotype, landrace Siberia, combined drought tolerance/adaptability with the largest total biomass at end of the recovery period, both in the irrigated control as in the drought stressed plants.

At the seedling stage, the drought resistance, drought recovery and drought adaptability indices calculated with total biomass were more associated to root growth than to shoot growth, as shown by the significant correlations with root biomass: $\operatorname{ResRB}(\mathrm{r}=0.79 * *), \operatorname{RecRB}\left(\mathrm{r}=0.78^{* *}\right)$ and AdapRB $\left(r=0.87^{* *}\right)$, respectively, while correlations with shoot biomass had lower values $(\operatorname{ResSB}, \mathrm{r}=0.74 * *$; RecSB, $r=0.45^{* *}$; AdapSB, $r=0.67^{* *}$, respectively). According to these results, at the seedling stage the root size is more determinant than the shoot size for drought stress tolerance/adaptability in maize seedlings. According to Villalobos-González et al. (2018), the changes in biomass allocation between root and shoot induced by water deficit, could be due to differences in turgor potential between leaves and roots (variables not measured in this study), since cell turgor affects growth rate both in root and shoot.

Even when the drought resistance and the subsequent recovery are both key determinants of drought adaptability 
in maize seedlings (Chen et al., 2016), the post-stress recovery seems to play the most important role in drought stress adaptability, because drought adaptability indices were more associated with drought recovery indices than with the drought resistance indices, based on the three measured variables (root biomass, shoot biomass and total biomass). Therefore, in drought tolerance/adaptability studies it is convenient to measure the recovery in order to get a more precise genotypic classification. For example, the genotype classification based solely on resistance index (ResTB) and by the amount of total biomass accumulated at end of drought stress, the maize landraces named Catorce, Delicias, Salero, Carpintería, SAnita, PAnteojitos, Berrones, Sabanilla and AraTrini, would have been selected as tolerant, even though they clearly behaved as drought susceptible at end of the recovery period.

Similarly, Perrone et al. (2012) reported that plants with greater drought recovery capacity have a greater chance of survival, particularly in locations with water deficits alternated and rewatering cycles, conditions that are typical in rainfed agricultural regions located in arid and semi-arid climates. Some physiological traits have been associated with recovering plant growth after a water deficit in maize, such as a rapid recovery of stomatal conductance, transpiration and assimilation rate (Chen et al., 2016), transcriptional coordination between leaves and roots after stress (Fan et al., 2013), antioxidant system (Xu et al., 2010), deeper roots, and more aquaporins in root cell membranes (Martre et al., 2002; Li et al., 2015).

In any case, a good performance at the beginning of the plant cycle (seedling stage) is essential to reach a good final performance and a high grain yield. However, a good seedling performance by itself is not enough to guarantee a good grain yield at the end of cycle. On the other hand, maize genotypes may behave differently in response to water deficits at a later plant growth stage.

\section{CONCLUSIONS}

In the maize landraces collected from Southern Nuevo Leon, Mexico, there is a wide genetic diversity in drought tolerance/adaptability at the seedling stage. The most tolerant genotypes were native GalTrini and SITexas because they presented the highest values in drought adaptability index (AdapBT) and because they accumulated more total biomass both during the drought stress period and afterwards during the recovery period. In contrast, the most susceptible genotypes were the landraces Berrones, Rodeo, Sabanilla, Carmen, and AraTrini, and the inbred line L65, which showed the lowest values in AdapBT and large losses in accumulated biomass that ranged between
52 and $65 \%$ at the end of recovery. The total biomass recorded before water stress (initial seedling vigor) was not related to the drought adaptability index. Root size was more determinant than shoot size for drought stress adaptability at the maize seedling stage. Among the three evaluated indices, the recovery index plays the most important role in drought stress adaptability of maize, followed by the drought resistance indices, for the three measured variables (root biomass, shoot biomass and total biomass). Therefore, it is convenient to measure the post stress recovery in drought tolerance studies in order to get more accurate genotypic classification.

\section{ACKNOWLEDGMENTS}

The authors are grateful to the National Council for Science and Technology (CONACYT) for providing the grant PN2016-3855 to support both the experimental execution and the doctoral scholarship for Eleazar Lugo Cruz. We also acknowledge the Colegio de PostgraduadosCampus Montecillo for providing the structural facilities (experimental field station, laboratory equipment) and additional financial resources for conducting this research.

\section{Author contributions}

Víctor A. González-Hernández, project leader, obtained the financial resources for the study execution, coordinated the whole research work, designed the experimental work and revised/edited the manuscript. Eleazar Lugo-Cruz, was a Ph.D. student, participated in the design of the experiment, carried out the experiment, analyzed the data and wrote the original draft. Leopoldo E. MendozaOnofre, collaborated in the experimental design, in data analysis and in the manuscript review. Amalio SantacruzVarela, reviewed the project and the manuscript. Francisco Zavala-García, reviewed the project and its execution. Ma. Alejandra Gutiérrez-Espinosa, proposed improvements to the project and participated in the critical review of this manuscript.

\section{REFERENCES}

Akinwale, R. O., F. E. Awosanmi, O. O. Ogunniyi and A. O. Fadoju. 2017. Determinants of drought tolerance at seedling stage in early and extra-early maize hybrids. Maydica. 62: 1-9.

Anjorin, F. B., S. A. Adejumo, K. S. and D. J. Ogunniyan. 2017. Seedling establishment, biomass yield and water use efficiencies of four maize varieties as influenced by water deficit stress. Cercet. Agron. Mold. 50: 21-34.

Aslam, M., M. A. Maqbool and R. Cengiz. 2015. Drought stress in maize (Zea mays L.): Effects, resistance mechanisms, global achievements and biological strategies for improvement. Springer Briefs in Agriculture, Springer International Publishing, London, p. 74.

Badr, A., H. H. El-Shazly, R. A. Tarawneh and A. Börner. 2020. 
Screening for drought tolerance in maize (Zea mays L.) germplasm using germination and seedling traits under simulated drought conditions. Plants. 9: 565.

Bashir, N., S. Mahmood, Z. Ullah, S. Rasul, H. Manzoor and H. Athar. 2016. Is drought tolerance in maize (Zea mays L.) cultivars at the juvenile stage maintained at the reproductive stage? Pak. J. Bot. 48: 1385-1392.

Belmont-Valadez, R. 2018. Sobre-Expresión de Rubisco activasa y de Dehidrina dhn1 en Plantas de Maíz por el Método Intragénico. Tesis de Licenciatura en Química Farmacéutica Biológica, Facultad de Química, Universidad Nacional Autónoma de México, México.

Chen, D., S. Wang, B. Cao, D. Cao, G. Leng, H. Li, L. Yin, L. Shan and $X$. Deng. 2016. Genotypic variation in growth and physiological response to drought stress and re-watering reveals the critical role of recovery in drought adaptation in maize seedlings. Front. Plant. Sci. 6: 1241.

Efeoğlu, B., Y. Ekmekçi and N. Çiçek. 2009. Physiological responses of three maize cultivars to drought stress and recovery. S. Afr. J. Bot. 75: 34-42.

Fan, X., G. Huang, L. Zhang, T. Deng and Y. Li. 2013. Adaptability and recovery capability of two maize inbred-line foundation genotypes, following treatment with progressive water-deficit stress and stress recovery. Agric. Sci. 4: 389-398.

Gil, M. A., P. A. López, O. A. Muñoz and H. S. López. 2004. Variedades criollas de maíz (Zea mays L.) en el estado de Puebla, México: Diversidad y utilización. In: J. L. Chávez-Servia, J. Tuxill and D. I. Jarvis (Eds.), Manejo de la diversidad de los cultivos en los agroecosistemas tradicionales. Instituto Internacional de Recursos Fitogenéticos. Cali, Colombia, pp. 18-25.

Hellin, J., M. R. Bellon and S. J. Hearne. 2014. Maize landraces and adaptation to climate change in Mexico. J. Crop. Impr. 28: 484-501.

Hernández-Guillén, A. K. 2016. Identificación de plantas de maíz que sobre-expresen genes marcadores de mejoramiento genético. Tesis de licenciatura en Química de Alimentos. Facultad de Química, Universidad Nacional Autónoma de México, México.

Hunt, R. 1990. Basic Growth Analysis Plant Growth Analysis for Beginners. Unwin Hyman Ltd., London.

INEGI. 2019. Instituto Nacional de Estadística y Geografía. Prontuario de Información Geográfica Municipal de los Estados Unidos Mexicanos y Mapa Digital. Instituto Nacional de Estadística y Geografía. Available from: http://www.gaia.inegi.org.mx/md m6/?v=bGF0OjIzLjMyMDA4LGxvbjotMTAxLjUwMDAwLHo6 MSxsOmMxMTFzZXJ2aWNpb3N8dGMxMTFzZXJ2aWNpb3M. [Last accessed on 2019 Jun 13].

Li, R., Y. Zeng, J. Xu, Q. Wang, F. Wu, M. Cao, H. Lan, Y. Liu and Y. Lu. 2015. Genetic variation for maize root architecture in response to drought stress at the seedling stage. Breed. Sci. 65: 298-307.
López-Santillán, J. A., S. Castro-Nava, C. Trejo-López, M. C. Mendoza-Castillo and J. Ortiz-Cereceres. 2004. Biomasa acumulada e intercambio gaseoso en maíz proveniente de semilla de diferente tamaño bajo humedad favorable y restringida. Phyton. 73: 243-248.

Mabhaudhi, T. and A. T. Modi. 2010. Early establishment performance of local and hybrid maize under two water stress regimes. S. Afr. J. Plant. Soil. 27: 299-304.

Martre, P., R. Morillon, F. Barrieu, G. B. North, P. S. Nobel and M. J. Chrispeels. 2002. Plasma membrane aquaporins play a significant role during recovery from water deficit. Plant. Physiol. 130: 2101-2110.

Munns, R. and R. A. Richards. 2007. Recent advances in breeding wheat for drought and salt stresses. In: M. A. Jenks, P. M. Hasegawa and M. S. Jain (Eds.), Advances in Molecular Breeding toward Drought and Salt Tolerant Crops. SpringerVerlag, Berlin, pp. 565-585.

Pawar, K. R., S. G. Wagh, P. P. Sonune, S. R. Solunke, S. B. Solanke, S. G. Rathod and S. N. Harke. 2020. Analysis of water stress in different varieties of maize (Zea mays L.) at the early seedling stage. Biotechnol. J. Int. 24: 15-24.

Perrone, I., C. Pagliarani, C. Lovisolo, W. Chitarra, F. Roman and A. Schubert. 2012. Recovery from water stress affects grape leaf petiole transcriptome. Planta. 235: 1383-1396.

Richards, R. A., G. J. Rebetzke, A. G. Condon and A. F. Herwaarden. 2002. Breeding opportunities for increasing the efficiency of water use and crop yield in temperate cereals. Crop. Sci. 42: 111-121.

SAS Institute. 2015. Statistical Analysis Software for Windows Version 9.4. SAS Institute, Cary, North Carolina, USA.

Teruel, M. E., C. A. Biasutti, M. C. Nazar and D. A. Peiretti. 2008. Efectos de aptitud combinatoria para vigor de plántula bajo estrés hídrico en maíz. Agriscientia. 25: 27-34.

Trachsel, S., D. Sun, F. M. San Vicente, H. Zheng, E. A. Suarez, R. Babu and X. Zhang. 2016. Identification of QTL for early vigor and stay-green conferring tolerance to drought in two connected advanced backcross populations in tropical maize (Zea mays L.). PLoS One. 11: e0163400.

Turner, N. C. 1979. Drought resistance and adaptation to water deficit in crop plants. In: H. Mussell and R. C. Staples, (Eds.), Stress physiology in crop plants. Wiley Interscience, New York, pp. 343-372.

Villalobos-González, A., C. López-Castañeda, S. Miranda-Colín, V. H. Aguilar-Rincón and M. B. López-Hernández. 2018. Efecto del estrés hídrico y nitrógeno en las raíces de variedades hibridas y criollas de maíz (Zea mays L.). Agroproductividad. 11: 3-8.

Xu, Z., G. Zhou and H. Shimizu. 2010. Plant responses to drought and rewatering. Plant. Signal. Behav. 5: 649-654. 